\title{
Biochemical characteristics of clinical and environmental isolates of Burkholderia pseudomallei
}

\author{
VANAPORN WUTHIEKANUN, M. D. SMITH, D. A. B. DANCE*, AMANDA L. WALSH, T. L. PITT† \\ and N. J. WHITE \\ Faculty of Tropical Medicine, Mahidol University, Rajvithi Road, Bangkok 10400, Thailand and Centre for \\ Tropical Medicine, Nuffield Department of Clinical Medicine, John Radcliffe Hospital, Oxford University, \\ Oxford OX3 9DU, * Public Health Laboratory, Derriford Hospital, Derriford Road, Plymouth PL6 8DH and \\ $\uparrow$ Laboratory of Hospital Infection, Central Public Health Laboratory, Colindale Avenue, London NW9 5HT
}

\begin{abstract}
The biochemical characteristics of 213 isolates of Burkholderia pseudomallei from patients with melioidosis and 140 isolates from the soil in central and northeastern Thailand were compared. Whereas the biochemical profiles of all the clinical isolates were similar, all soil isolates from the central area and $25 \%$ of isolates from northeastern Thailand comprised a different phenotype. This was characterised by the ability to assimilate L-arabinose $(100 \%)$, adonitol $(100 \%)$, 5-keto-gluconate $(90 \%)$ and D-xylose $(84 \%)$, but failure to assimilate dulcitol $(0 \%)$, erythritol $(0 \%)$ and trehalose (10\%). Compared with clinical isolates, these organisms had similar antibiotic susceptibility profiles and were also recognised by a specific polyclonal antibody against $B$. pseudomallei. As melioidosis is rare in central Thailand, but common in the northeast, this raises the possibility that this biochemical phenotype may be less virulent, or may even represent a different species.
\end{abstract}

\section{Introduction}

Burkholderia (formerly Pseudomonas) pseudomallei is an environmental saprophyte capable of causing devastating systemic infections in many species of mammals and birds. Although B. pseudomallei has been isolated from environmental samples across the tropical world, the disease in man is confined to the endemic areas of eastern Asia and northern Australia [1]. In northeastern Thailand B. pseudomallei is a major cause of morbidity and mortality, causing onefifth of all community-acquired septicaemias [2]. However, B. pseudomallei is also isolated readily from soil samples in the southern and central regions of Thailand, where clinical disease is rare $[3,4]$. To investigate this discrepancy between the distribution of disease and the distribution of the organism in the environment, this study compared the biochemical and antigenic features, and antibiotic susceptibilities, of clinical and environmental isolates of $B$. pseudomallei.

Received 25 Jan. 1996; accepted 26 March 1996. Corresponding author: Dr N. J. White.

\section{Materials and methods}

\section{Bacterial isolates}

Clinical and laboratory studies on melioidosis have been conducted in Ubon Ratchatani, northeast Thailand, since 1986. More than 1100 patients with cultureproven melioidosis have been seen during this time. Single isolates of $B$. pseudomallei from 213 of these patients were used for this study.

The soil isolates of $B$. pseudomallei were selected from those collected in a survey of rice paddies in northeastern Thailand [5] and in a quantitative comparison of the isolation of the organism from soil in central and northeastern Thailand [4]. A maximum of two isolates was selected from each site sampled, giving a total of 140 isolates, 115 from the northeast and 25 from the central region.

\section{Organism identification}

All isolates were identified initially by their characteristic colonial morphology on a differential agar medium [6], positive oxidase reaction, and resistance to colistin and gentamicin [7]. 
Biochemical identification was obtained with API 20NE (bioMérieux) following the manufacturer's instructions, as detailed previously [7].

\section{Extended biochemical characteristics}

For 100 strains of B. pseudomallei further biochemical profiles were obtained with API $50 \mathrm{CH}$ (bioMérieux) following the manufacturer's guidelines. The 49 individual carbohydrates tested are given in Table 2. The medium used was a sterile minimal salts solution (ammonium chloride $20 \mathrm{~g}$, ammonium nitrate $4 \mathrm{~g}$, anhydrous sodium sulphate $8 \mathrm{~g}$, dipotassium hydrogen orthophosphate $12 \mathrm{~g}$, potassium dihydrogen orthophosphate $4 \mathrm{~g}$, magnesium sulphate $0.4 \mathrm{~g}$, all from Sigma, and distilled water to $1 \mathrm{~L}$ ) to which agar $0.15 \%$ was added. The ability to assimilate a single carbohydrate was recorded if there was any growth after incubation for $48 \mathrm{~h}$ at $37^{\circ} \mathrm{C}$.

\section{Arabinose agar}

A single carbohydrate agar was used for L-arabinose and glucose assimilation with a larger number of isolates of $B$. pseudomallei. This consisted of minimal salts solution $(25 \mathrm{ml})$, agar $2 \%$ solution $(75 \mathrm{ml})$ and carbohydrate (L-arabinose or glucose; Sigma) $10 \%$ solution $(2 \mathrm{ml})$. Each solution was sterilised separately and then mixed together. The agar plates were spotted with $3 \mu \mathrm{l}$ of a 1 in 10 dilution of an overnight bacterial suspension adjusted to a 0.5 MacFarland standard. Results were recorded as growth after incubation for $48 \mathrm{~h}$ at $37^{\circ} \mathrm{C}$. The glucose agar was used as a growth control for all isolates.

\section{Antigenicity}

The antigenic similarity of clinical and soil isolates was checked with a latex agglutination test for the rapid identification of $B$. pseudomallei, as described previously [8]. The latex particles were coated with a rabbit antiserum to boiled whole cells of a clinical isolate (NCTC 1688) of B. pseudomallei.

\section{Antibiotic susceptibilities}

Tests for susceptibility to ceftazidime, chloramphenicol, co-amoxiclav and doxycycline were performed by disk diffusion [9].

\section{Statistical analysis}

Proportions were compared with the $\chi^{2}$-squared test or Fisher's exact test.

\section{Results}

\section{Arabinose assimilation}

The API 20NE results of 213 clinical isolates and 69 soil isolates of $B$. pseudomallei are shown in Table 1. There was a highly significant difference between the two groups in their ability to assimilate arabinose. None of the clinical isolates could assimilate arabinose whereas $52 \%$ of the soil isolates did so. Within the soil group there was significant geographical variation; all 25 central region isolates assimilated arabinose but only $11(25 \%)$ of the 44 northeastern isolates $(p<0.00001)$. There was also a small difference in the assimilation of maltose $(7.5 \%$ versus $0 \%$ of clinical

Table 1. Comparison of API 20NE biochemical reactions of clinical and soil isolates of $B$. pseudomallei

\begin{tabular}{lccl}
\hline & \multicolumn{2}{c}{ Percentage positive } & \\
\cline { 2 - 3 } Substrate & $\begin{array}{c}\text { Clinical isolates } \\
(\mathrm{n}=213)\end{array}$ & $\begin{array}{c}\text { Soil isolates } \\
(\mathrm{n}=69)\end{array}$ & $\mathrm{p}$ value \\
\hline Nitrate & 100 & 100 & \\
Tryptophan & 100 & 100 & \\
Glucose (acidification) & 0 & 0 & \\
Arginine & 100 & 100 & 0.35 \\
Urea & 0 & 0 & \\
Aesculin & 22.1 & 27.5 & \\
Gelatin & 100 & 100 & \\
PNPG & 0 & 0 & \\
Assimilation of: & & & \\
Glucose & 100 & 100 & 0.00001 \\
Arabinose & 0 & 52.2 & \\
Mannose & 100 & 100 & \\
Mannitol & 100 & 100 & \\
N-acetylglucosamine & 99.6 & 100 & \\
Maltose & 7.5 & 0 & \\
Gluconate & 99.2 & 100 & \\
Caprate & 100 & 100 & \\
Adipate & 100 & 100 & \\
Malate & 100 & 100 & \\
Citrate & 92.9 & 97.1 & \\
Phenylacetate & 96.7 & 92.8 & \\
\hline
\end{tabular}


and soil isolates, respectively) but all other reactions gave very similar results.

Assimilation of arabinose in the API 20NE system was then compared with growth on L-arabinose agar for 58 of the soil isolates (half of which assimilated arabinose in API 20NE). The results were identical. Isolates with the ability to assimilate arabinose in API $20 \mathrm{NE}$ or grow on L-arabinose agar are designated ara ${ }^{+}$ and isolates without this ability are designated $\mathrm{ara}^{-}$.

Subsequently, growth on L-arabinose agar was assessed for another 71 isolates. Eleven isolates were assessed by API $20 \mathrm{NE}$ only. The results of L-arabinose assimilation by agar and API were then combined. Of the northeastern isolates, $29(25 \%)$ of 115 were $\mathrm{ara}^{+}$ and $86(75 \%)$ were ara $^{-}$. In contrast, all 25 central region isolates were $\operatorname{ara}^{+}(\mathrm{p}<0.00001)$.

In northeastern Thailand both $\mathrm{ara}^{+}$and $\mathrm{ara}^{-}$soil isolates of $B$. pseudomallei may be found in the same location. During a previous environmental survey [5], a detailed study of one rice farm was performed. Of 89 isolates with API 20NE results, $69(77.5 \%)$ were $\mathrm{ara}^{+}$and $20(22.5 \%)$ were ara $^{-}$.

\section{Extended biochemical characteristics}

Extended biochemical profiles were obtained for 100 isolates of B. pseudomallei by the API $50 \mathrm{CH}$ system. Fifty of these isolates did not assimilate arabinose in
API 20NE ( $\mathrm{ara}^{-} ; 25$ clinical isolates and 25 soil isolates from the northeastern region) and 50 isolates assimilated arabinose in API 20NE ( $\mathrm{ara}^{+} ; 25$ soil isolates from the central region and 25 isolates from the northeastern region). The results are shown in Table 2 . The most significant differences were that $\mathrm{ara}^{+}$ isolates of $B$. pseudomallei assimilated L-arabinose $(100 \%)$, adonitol $(100 \%)$, 5-keto-gluconate $(90 \%)$ and D-xylose $(84 \%)$, but failed to assimilate dulcitol $(0 \%)$, erythritol $(0 \%)$ and trehalose $(10 \%)$. Smaller, but still significant, differences were found in the ability to assimilate aesculin, D-arabinose, L-arabitol, glycerol, glycogen, starch, sucrose and xylitol. Within the ara ${ }^{-}$ group, there were no significant differences between clinical and soil isolates. Comparing soil $\mathrm{ara}^{+}$isolates, there were small but significant differences between central and northeastern isolates in their ability to assimilate sucrose $(8 \%$ versus $36 \% ; p=0.04)$ and 5 keto-gluconate $(80 \%$ versus $100 \% ; \mathrm{p}=0.025)$.

\section{Antigenicity}

All 140 soil isolates of $B$. pseudomallei $\left(86 \mathrm{ara}^{-}\right.$and $54 \mathrm{ara}^{+}$) gave a positive reaction in the latex agglutination test. The majority of clinical isolates included in this study were not tested for latex agglutination. However, the latex agglutination test was evaluated initially with 52 clinical isolates of $B$. pseudomallei [8] and no false positive reactions were recorded or have been seen subsequently in 4 years of use in clinical studies.

Table 2. Extended biochemical reactions of $B$. pseudomallei to compare ara ${ }^{-}$ clinical or soil isolates with ara $^{+}$soil isolates tested in API $50 \mathrm{CH}$

\begin{tabular}{|c|c|c|c|}
\hline \multirow[b]{2}{*}{ Substrate* } & \multicolumn{2}{|c|}{ API $20 \mathrm{NE}$ arabinose assimilation } & \multirow[b]{2}{*}{$\mathrm{p}$ value } \\
\hline & $\begin{array}{c}\text { Negative }(\%) \\
\quad(n=50)\end{array}$ & $\begin{array}{l}\text { Positive }(\%) \\
(n=50)\end{array}$ & \\
\hline Glycerol & 80 & 98 & 0.01 \\
\hline Erythritol & 88 & 0 & $<0.00001$ \\
\hline D-Arabinose & 36 & 0 & 0.00001 \\
\hline L-Arabinose & 0 & 100 & $<0.00001$ \\
\hline Ribose & 2 & 0 & \\
\hline D-Xylose & 0 & 84 & $<0.00001$ \\
\hline L-Xylose & 22 & 14 & 0.3 \\
\hline Adonitol & 4 & 100 & $<0.00001$ \\
\hline L-Sorbose & 2 & 0 & \\
\hline Rhamnose & 2 & 0 & \\
\hline Dulcitol & 98 & 0 & $<0.00001$ \\
\hline $\mathrm{N}$-acetylglucosamine & 98 & 98 & \\
\hline Aesculin & 0 & 30 & 0.0001 \\
\hline Sucrose & 2 & 22 & 0.006 \\
\hline Trehalose & 98 & 10 & $<0.00001$ \\
\hline Starch & 46 & 98 & 0.00001 \\
\hline Glycogen & 44 & 100 & $<0.00001$ \\
\hline Xylitol & 94 & 72 & 0.008 \\
\hline L-Fucose & 94 & 96 & \\
\hline L-Arabitol & 82 & 100 & 0.002 \\
\hline 5-Keto-gluconate & 0 & 90 & $<0.00001$ \\
\hline
\end{tabular}

${ }^{*}$ Carbohydrates assimilated by all 100 isolates of $B$. pseudomallei: galactose, D-glucose, Dfructose, D-mannose, inositol, mannitol, sorbitol, D-arabitol, gluconate and 2-keto-gluconate. Carbohydrates not assimilated by any isolate of $B$. pseudomallei: $\beta$-methyl-D-xyloside, $\alpha$-methylD-mannoside, $\alpha$-methyl-D-glucoside, amygdalin, arbutin, salicin, cellobiose, maltose, lactose, melibiose, inulin, melezitose, D-raffinose, $\beta$-gentiobiose, D-turanose, D-lyxose, D-tagatose and Dfucose. 


\section{Antibiotic susceptibilities}

All 140 soil isolates of B. pseudomallei ( $86 \mathrm{ara}^{-}$and $54 \mathrm{ara}^{+}$) were susceptible, according to the NCCLS definitions [9], to the four antibiotics tested. Of the 213 clinical isolates of B. pseudomallei, 202 were susceptible to all four antibiotics. Ten patients had isolates that were resistant to chloramphenicol alone; nine were resistant when the patient was admitted to hospital and one developed resistance during treatment. The remaining patient's isolate was resistant to co-amoxiclav, but this also developed during treatment.

\section{Discussion}

Clinical and environmental isolates of $B$. pseudomallei are morphologically similar and antigenically indistinguishable. The immunodominant antigen of $B$. pseudomallei is the cell wall lipopolysaccharide [10]. This is remarkably invariant among different isolates, and is sufficiently similar in clinical and environmental isolates for all to be recognised by the polyclonal antibody used for the latex agglutination test. The antibiotic susceptibility profile of clinical isolates of $B$. pseudomallei, characterised by aminoglycoside resistance and susceptibility to ceftazidime, co-amoxiclav, chloramphenicol and tetracyclines was also found in the soil isolates.

In terms of biochemical reactions there were significant differences between environmental and clinical isolates. Obviously organisms causing clinical melioidosis originate from the soil, and organisms were found in the soil of northeastern Thailand that were identical in all respects with those causing disease. However, another discrete biotype was also present. This biotype is characterised by assimilation of Larabinose $(100 \%)$, adonitol $(100 \%)$, 5-keto-gluconate $(90 \%)$ and D-xylose $(84 \%)$, but failure to assimilate dulcitol $(0 \%)$, erythritol $(0 \%)$ and trehalose $(10 \%)$. This soil biotype (designated ara ${ }^{+}$) was found together with the clinical biotype (designated ara $^{-}$) in ricepaddies of the northeast, but was the only type isolated in the central area of Thailand. This corresponds with the clinical distribution of melioidosis. The infection is common in the northeast, where it causes c. $20 \%$ of community-acquired septicaemias, but is rare in the central region, yet rice-farming practices (the major occupational risk factor for the disease) are common to both regions. This suggests that the ara ${ }^{+}$biotype may be considerably less virulent than the ara $^{-}$biotype.

This difference in biochemical reactivity, and possible difference in virulence raises the possibility that these two biotypes are sufficiently far apart to constitute different species. $\mathrm{Ara}^{+}$soil isolates of $B$. pseudomallei are significantly different from other L-arabinosepositive environmental Burkholderia species described by Gillis et al. [11] and Yabuuchi et al. [12]. B. cepacia, B. gladioli and B. pickettii are arginine dihydrolase-negative and have significant differences in the assimilation of nine or more of the carbohydrates. B. andropogonis, B. caryophylli, B. glumae and $B$. plantarii differ from ara $^{+} B$. pseudomallei in the assimilation of seven or eight carbohydrates. $B$. cocovenenans and $B$. phenazinium differ by assimilation of five and four carbohydrates respectively. Compared with $\operatorname{ara}^{+} B$. pseudomallei, B. vandii [13] differs in the ability to assimilate adonitol, lactose and starch, but only one isolate and few carbohydrates were tested. However, B. vandii is susceptible to gentamicin. Recently a new species of Burkholderia, $B$. vietnamensis, was isolated from rice rhizopheres in southern Vietnam [11]. This species also differs from the $\mathrm{ara}^{+}$isolates of $B$. pseudomallei in the present study in that the former does not produce arginine dihydrolase and fails to assimilate adonitol, L-arabitol, glycogen and starch. Also, B. vietnamensis assimilates D-arabinose, cellobiose, dulcitol, gentiobiose, D-lyxose and D-fucose.

The results for ara $^{-}$isolates of $B$. pseudomallei differ significantly from those of Redfearn et al. [14] in the ability to assimilate cellobiose, D-fucose, maltose, salicin and sucrose, but this may reflect differences in methodology and geographical distribution of the isolates.

The absence of disease caused by the ara ${ }^{+}$biotype of B. pseudomallei should now be confirmed by a comparison of virulence in an animal challenge. If lack of virulence is confirmed this would also raise the possibility that an apparently immunologically similar organism might be a vaccine candidate for the prevention of melioidosis, and might provide an important insight into the mechanisms of virulence in B. pseudomallei.

It is also possible that the ara ${ }^{+}$biotype represents a new species of Burkholderia which is morphologically and antigenically similar to $B$. pseudomallei. Therefore further study and comparison of the genetic structure of the two biotypes is needed.

We thank $\mathrm{Mr} \mathrm{S}$. Langla for technical assistance, and $\mathrm{Dr} \mathrm{Y}$. Supputamongkol for help in collecting clinical isolates. We are grateful to Dr Wipada Chaowagul of Sappasitprasong Hospital, Ubon Ratchatani, for her support and guidance. This study was part of the Wellcome-Mahidol University, Oxford Tropical Medicine Research Programme, funded by the Wellcome Trust of Great Britain.

\section{References}

1. Dance DAB. Melioidosis: the tip of the iceberg? Clin Microbiol Rev 1991; 4: 52-60.

2. Chaowagul W, White NJ, Dance DAB et al. Melioidosis: a major cause of community-acquired septicemia in Northeastern Thailand. J Infect Dis 1989; 159: 890-899.

3. Nachiangmai N, Patamasucon P, Tipayamonthien B, Kongpon A, Nakaviroj S. Pseudomonas pseudomallei in southern Thailand. Southeast Asian J Trop Med Public Health 1985; 16: $83-87$. 
4. Smith MD, Wuthiekanun V, Walsh AL, White NJ. Quantitative recovery of Burkholderia pseudomallei from soil in Thailand. Trans R Soc Trop Med Hyg 1995; 89: 488-490.

5. Wuthiekanun V, Smith MD, Dance DAB, White NJ. Isolation of Pseudomonas pseudomallei from soil in north-eastern Thailand. Trans $R$ Soc Trop Med Hyg 1995; 89: 41-43.

6. Ashdown LR. An improved screening technique for isolation of Pseudomonas pseudomallei from clinical specimens. Pathology 1979; 11: 293-297.

7. Dance DAB, Wuthiekanun V, Naigowit P, White NJ. Identification of Pseudomonas pseudomallei in clinical practice: use of simple screening tests and API 20NE. J Clin Pathol 1989; 42: 645-648

8. Smith MD, Wuthiekanun V, Walsh AL, Pitt TL. Latex agglutination test for the identification of Pseudomonas pseudomallei. J Clin Pathol 1993; 46: 374-375.

9. National Committee for Clinical Laboratory Standards. Performance standards for antimicrobial disk susceptibility tests, 3rd edn; approved standard M2-A3. National Committee for Clinical Laboratory Standards, Villanova, PA. 1984
10. Pitt TL, Aucken H, Dance DAB. Homogeneity of lipopolysaccharide antigens in Pseudomonas pseudomallei. $J$ Infect 1992; 25: 139-146.

11. Gillis M, Van TV, Bardin R et al. Polyphasic taxonomy in the genus Burkholderia leading to an emended description of the genus and proposition of Burkholderia vietnamensis sp. nov. for $\mathrm{N}_{2}$-fixing isolates from rice in Vietnam. Int $J$ Syst Bacteriol 1995; 45: 274-289.

12. Yabuuchi E, Kosako Y, Oyaizu H et al. Proposal of Burkholderia gen. nov. and transfer of seven species of the genus Pseudomonas homology group II to the new genus, with the type species Burkholderia cepacia (Palleroni and Holmes 1981) comb. nov. Microbiol Immunol 1992; 36: 1251-1275.

13. Urakami T, Ito-yoshida C, Araki H, Kijima T, Suzuki K, Komagata K. Transfer of Pseudomonas glumae to Burkholderia as Burkholderia spp. and description of Burkholderia vandii sp. nov. Int $J$ Syst Bacteriol 1994; 44: 235-245.

14. Redfearn MS, Palleroni NJ, Stanier RY. A comparative study of Pseudomonas pseudomallei and Bacillus mallei. $J$ Gen Microbiol 1966; 43: 293-313. 